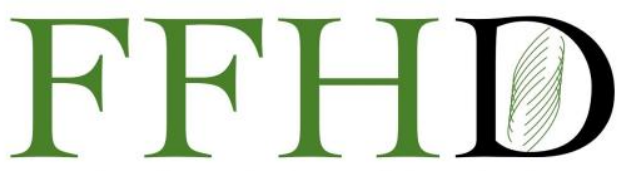

Functional Foods in Health and Disease

\title{
Moriche Palm (Aguaje) Extract improves indefinite complaints in Japanese females: a randomized, placebo-controlled, double-blind trial
}

\author{
Tsuyoshi Takara', Kazuo Yamamoto², Naoko Suzuki², Shinichiro Yamashita², Shinichiro lio ${ }^{2}$, \\ Hayata Noguchi' ${ }^{2}$, Toshihiro Kakinuma², Asami Baba², Saya Yamamoto ${ }^{3}$, Toshio Morikawa ${ }^{3}$, \\ Shogo Takeda ${ }^{4}$, Hiroshi Shimoda4*
}

\begin{abstract}
${ }^{1}$ Takara Clinic, Medical Corporation Seishinkai, 9F Taisei Building, 2-3-2, Higashi-Gotanda, Shinagawa-ku, Tokyo 1410022, Japan; '2ORTHOMEDICO Inc., 2F Sumitomo Fudosan Korakuen Bldg., 1-4-1 Koishikawa Bunkyo-ku, Tokyo, 1120002 Japan; ${ }^{3}$ Pharmaceutical Research and Technology Institute, Kindai University, 3-4-1 Kowakae, Higashi-Osaka, Osaka 577-8502, Japan; ${ }^{4}$ Research and Development Division, Oryza Oil and Fat Chemical Co., Ltd., 1 Numata, Kitagata-cho, Ichinomiya, Aichi 493-8001, Japan
\end{abstract}

*Corresponding author: Hiroshi Shimoda, Ph.D, Research \& Development Division, Oryza Oil \& Fat Chemical Co., Ltd., 1 Numata, Kitagata-cho, Ichinomiya, Aichi 493-8001, Japan.

Submission Date: August $5^{\text {th }}, 2020$; Acceptance Date: September $4^{\text {th }}, 2020$; Publication Date: September 16, 2020

Please cite this article as: Takara T., Yamamoto K., Suzuki N., Yamashita S., lio S., Noguchi H., Kakinuma T., Baba. Moriche Palm (Aguaje) Extract Improves Indefinite Complaints in Japanese Females: A Randomized, Placebocontrolled, Double-blind Trial. Functional Foods in Health and Disease 2020; 10(9): 379-396. DOI: https://www.doi.org/10.31989/ffhd.v10i9.742

\footnotetext{
ABSTRACT

Background and objective: The fruit of Mauritia flexuosa (moriche palm), which is known as "Aguaje," has been used for beverages and processed foods. Recently, we found that several methoxyflavans are contained in the fruit and they exhibit estrogenic activities. Therefore, moriche palm extract (MPE) may function as a phytoestrogen and improve the symptoms induced by estrogen deficiency. However, the clinical effects of MPE on females has not yet been reported. We conducted a clinical trial of MPE on undefined complaints related to premenstrual syndrome (PMS) in healthy Japanese females.
} 


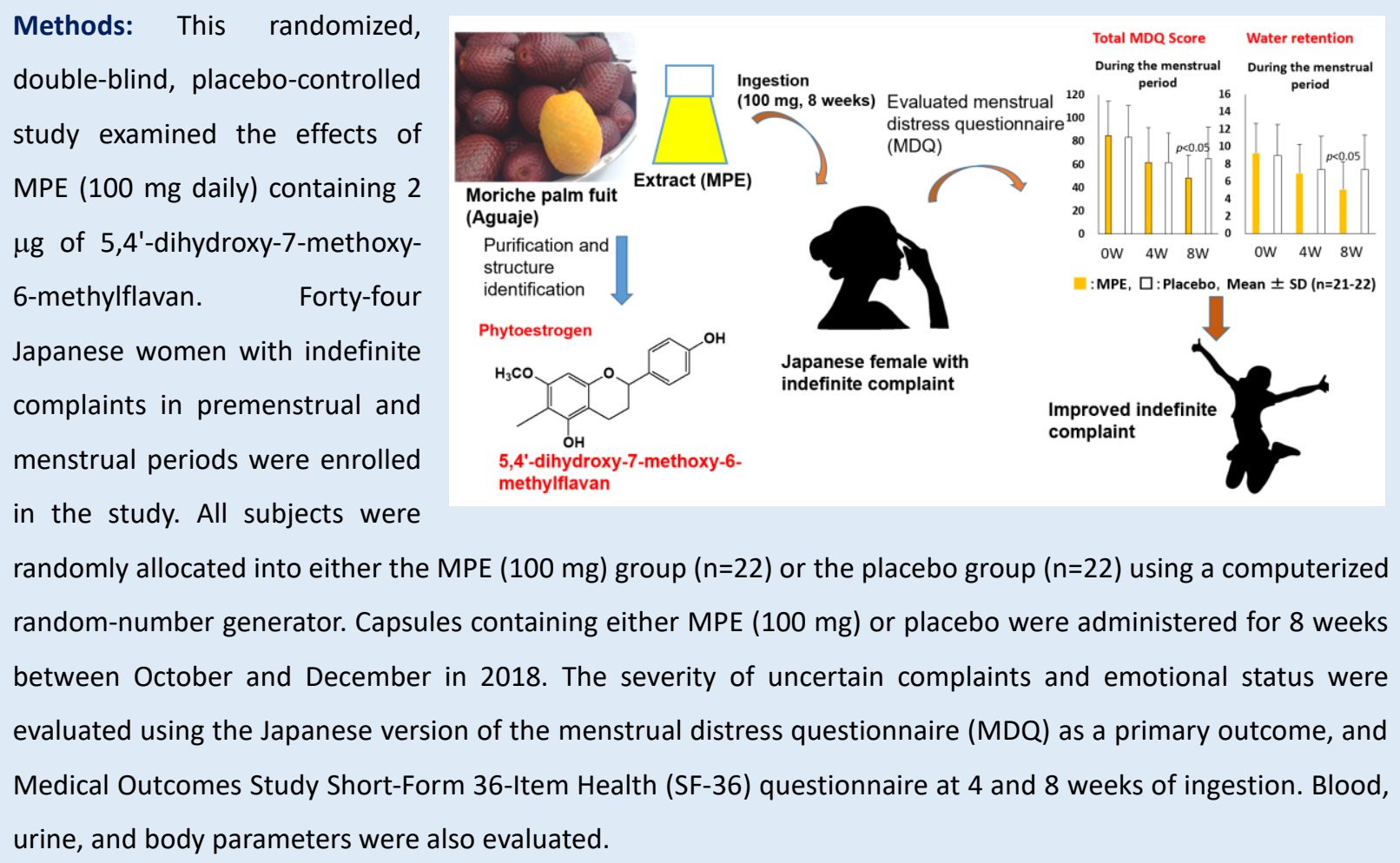

Moriche palm fuit Extract (MPE) (Aguaje)

Purification and structure identification

Phytoestrogen

randomly allocated into either the MPE $(100 \mathrm{mg})$ group $(n=22)$ or the placebo group $(n=22)$ using a computerized random-number generator. Capsules containing either MPE (100 mg) or placebo were administered for 8 weeks between October and December in 2018. The severity of uncertain complaints and emotional status were evaluated using the Japanese version of the menstrual distress questionnaire (MDQ) as a primary outcome, and Medical Outcomes Study Short-Form 36-Item Health (SF-36) questionnaire at 4 and 8 weeks of ingestion. Blood, urine, and body parameters were also evaluated.

Results: Forty-three subjects completed the trial, and the per protocol set comprised 21 subjects in the MPE (100 $\mathrm{mg}$ group and 22 subjects in the placebo group. After ingesting MPE for 4 weeks, arousal in the premenstrual period significantly improved in the MPE (100 mg) group. After 8 weeks, the summary score, water retention, impaired concentration and control during menstrual period significantly improved in the MPE (100 mg) group. Contrarily, among SF-36 domain scores, significant ameliorating effects of MPE were not observed compared with those of the placebo group. Laboratory tests revealed no abnormalities suggesting adverse effects of MPE.

Conclusions: MPE (100 mg/day for 8 weeks) improved several indefinite complaint parameters related to mensuration. MPE was suggested to be useful for improving anxiety related to PMS.

Keywords: Menstrual distress questionnaire; SF-36 questionnaire; moriche palm; methoxyflavan; indefinite complaint

CFFC 2020. This is an Open Access article distributed under the terms of the Creative Commons Attribution 4.0 License (http://creativecommons.org/licenses/by/4.0)

\section{BACKGROUND}

The fruit of Mauritia flexuosa (moriche palm) is cultivated in the Amazonian area of Peru and Brazil. The oval fruit is covered with a scaly dark red peel and contains a large seed. The edible part is yellow to orange because it contains carotenoids. The taste is like a steamed sweet potato. The fruit paste diluted with water is a popular beverage in the region. The fruit is also used for processed foods such as popsicles, cooking oil, and dietary supplements. It is called "Aguaje" in 
Peru, and is said to enlarge the breasts and is considered to contain phytoestrogens. However, the active phytoestrogen has not been identified. Recently, we found that methoxyflavans, including 5,4'dihydroxy-7-methoxy-6-methylflavan, in moriche palms exhibit estrogenic activity and hypothesized that compounds are involved in the breast enlargement effects of moriche palm. There are several reports of methoxyflavans isolated from palm plants such as sago palms [1] and Chinese dragon's blood [2]. On the other hand, diverse biological effects of methoxyflavans have been reported, including osteogenic [3], antiinflammatory [4, 5], anti-androgenic [6], lipase inhibitory [7] and nitric oxide inhibitory activities [8]. Among them, osteogenic and anti-androgenic effects of methoxyflavans $[3,6]$ suggested that the moriche palm containing methoxyflavans exhibits estrogenic activity via phytoestrogens.

One of the important effects of phytoestrogens is the improvement of female-specific symptoms caused by estrogen deficiency in the premenstrual period (PMS) [9] and postmenopausal period [10]. Soy isoflavones and a metabolite of daidzein, (S)-equol, reduced the risk of cardiovascular disease [11], osteoporosis [10,12] and anxiety [13] in premenopausal women. Although there are few reports of soy isoflavones in young women, it ameliorates physical premenstrual syndromes such as headaches and breast tenderness [14]. In addition, traditionally used medicinal herbs, such as fennel seeds [15], roots of Pueraria candollei var. mirifica [16], grape-derived resveratrol [17] and fenugreek seeds [18], for symptoms in the menopausal period were confirmed to be effective for unidentified female complaints.

Based on these reports and moriche palms containing methoxyflavans as phytoestrogen, it was predicted that moriche palm extract (MPE) can ameliorate symptoms caused by estrogen deficiency. Therefore, a clinical trial was conducted using healthy Japanese adults to investigate this possibility.

\section{MATERIALS AND METHODS}

Identification and evaluation of estrogenic compound in MPE: Dried moriche palm fruit pulp was obtained from Peru through Ajuto Inc. (Tokyo, Japan). The powdered pulp (100 kg) was extracted with mixture of n-hexane: $\operatorname{EtOH}(9: 1,400 \mathrm{~L})$ at $40^{\circ} \mathrm{C}$ for $2 \mathrm{hr}$ and the solvent was evaporated. Obtained extract $(6.2 \mathrm{~kg}$, yield: $6.2 \%)$ as moriche palm extract (MPE).

For isolation of 5,7-dihydroxy-4'-methoxy-6methylflavan, moriche palm oil (Mcassab comércio Eindústria Ltda, Brazil, 5 kg) was diluted in n-hexane (10 $\mathrm{kg}$ ) and added $70 \% \mathrm{EtOH}(10 \mathrm{~kg})$. The mixture was mixed and portioned to obtain the lower layer. Then, 70\% EtOH (10 kg) was added to $\mathrm{n}$-hexane layer and the portion procedure was repeated. The obtained aqueous EtOH (lower) layer was evaporated to obtain aqueous EtOH portion (3.75 g). The portion was separated by flash silica gel column chromatography (ODS $2 \mathrm{~L}$, Yamazen Co. Ltd., Osaka, Japan) with mixture of acetone: $\mathrm{MeOH}: \mathrm{H}_{2} \mathrm{O}$ (3: 3: 4). Flow late was $15 \mathrm{~mL} / \mathrm{min}$ and detection wavelength was $210 \mathrm{~nm}$ to obtain fraction (Fr). 1 (374 mf), Fr. 2 (478 mg), Fr. 3 (884 mg) and Fr. 4 (1.87 g). Fr. 2 was repeatedly purified by HPLC (Inertsil Ph-3, 20ф× 250 mm, GL Science Co. Inc., Tokyo, Japan) with mixture of acetone: $\mathrm{MeOH}: \mathrm{H}_{2} \mathrm{O}(3: 3: 4)$ to obtain 5,7-dihydroxy-4'-methoxy-6-methylflavan (38.4 $\mathrm{mg})$. The chemical structure was determined by ${ }^{1} \mathrm{H}$ - and ${ }^{13} \mathrm{C}-\mathrm{NMR}$ spectra with the referenced values listed in the previous report [2].

Estrogenic activity was evaluated by MCF-7 proliferation assay [19]. Sub-cultured MCF-7 cells (2,000 cells $/ 100 \mu \mathrm{L}$ ) were suspended in phenol red-free DMEM containing 5\% charcoal/dextran-treated FBS and 
penicillin (100 units $/ \mathrm{ml})$ and streptomycin $(100 \mu \mathrm{g} / \mathrm{ml})$ mixture, seeded into a 96-well culture plate, and incubated for $24 \mathrm{hr}$. Then, a test sample solution dissolved in dimethyl sulfoxide (DMSO) and diluted in medium (11 $\mu \mathrm{L}$ ) was added to the wells and the cells were cultured for 4 days. Subsequently, MTT solution (5 $\mathrm{mg} / \mathrm{mL}$ in PBS) was added (10 $\mu \mathrm{L})$ and incubation was continued for $4 \mathrm{hr}$. After removing the medium, $40 \mathrm{mM}$ $\mathrm{HCl}$ in isopropanol (100 $\mu \mathrm{L})$ was added to dissolve the formazan product and absorbance of the formazan solution was measured with at $570 \mathrm{~nm}$ referenced at $660 \mathrm{~nm}$.

Participants and grouping: All subjects were recruited between July $20^{\text {th }}$ and September $8^{\text {th }}, 2018$ through the Go106 website (https://www.go106.jp/) operated by ORTHOMEDICO Inc. (Tokyo, Japan). The inclusion criteria were healthy Japanese female adults with indefinite complaints in the premenstrual and during menstrual periods. Exclusion criteria were as follows:

1) Current or previous cancer, heart failure, or myocardial infarction.

2) Current treatment for arrhythmia, hepatitis, nephritis, rheumatoid arthritis, cerebrovascular disease, diabetes, hyperlipidemia, hypertension, or other chronic diseases.

3) Diagnosed PMS or premenstrual dysphoric disorder (PMDD).

4) Current use of medications or dietary supplements.

5) Menopausal women.

6) Subjects with allergic reactions to foods related to moriche palms or medicines.

7) Pregnancy, lactation or expected/planned pregnancy during the study period.
8) Subjects currently participating in another clinical trial or who had participated within the previous 3 months.

9) Subjects determined to be inappropriate for the study for other reasons by the attending physician.

10) Having counseling or psychotherapy.

11) Taking hormonal therapy.

12) Diagnosed or previously diagnosed with psychiatric disorders.

Forty-four subjects with relatively high total scores on the menstrual distress questionnaire (MDQ) [20] were selected after they were confirmed to be suitable for the study by a physician (Fig. 1). The subjects were asked to avoid excessive eating and drinking, and were requested to maintain a regular lifestyle during the study period. One day before testing, subjects were required to avoid excessive drinking of alcohol and strenuous exercise, and they fasted for 6 hours; water intake was allowed; prior to blood collection.

The sample size (number of subjects) was determined based on the following: The number of subjects was designed under the assumption that the effect size $(d)$ due to the effects of the intervention of this study on the MDQ total score was 0.9. As the number of subjects per group was 20 when the significance level $(\alpha)$ was $5 \%$ and the detection power (1- 6 ) was $80 \%$, the target number of subjects was 40 . The dropout rate during participation in the study was expected to be approximately $10 \%$, and it was decided to increase the number of participants by 2 in each group, and the number of subjects to be included was 44. 


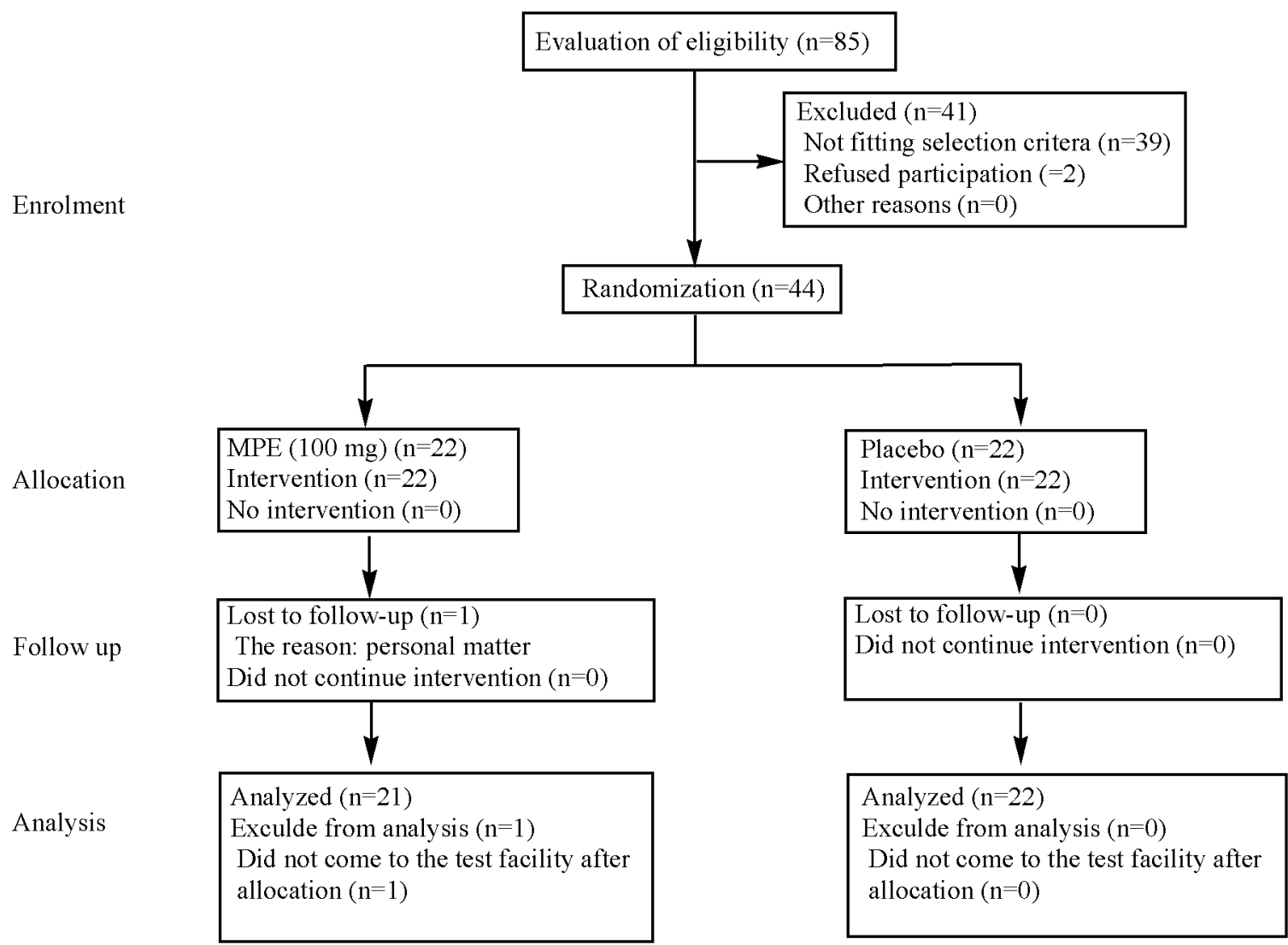

Figure 1. Flowchart showing the characteristics of the subjects.

Preparation and allocation of test samples: The test samples (indistinguishable brown capsules containing either MPE or placebo) were provided by Oryza Oil \& Fat Chemical Co., Ltd. as hard capsules. The MPE capsules contained $50 \mathrm{mg}$ of Aguaje Extract (standardized moriche palm extract containing $20 \mu \mathrm{g} / \mathrm{g}$ of 5,4'-dihydroxy-17'-methoxy-6-methylflavan) and 50 $\mathrm{mg}$ of dextrin. Aguaje Extract consisted of 99\% MPE and $1 \%$ mixed tocopherols. The placebo capsules contained $100 \mathrm{mg}$ of dextrin and $0.5 \mathrm{mg}$ of mixed tocopherols. The contents of 5,4'-dihydroxy-7-methoxy-6-methylflavan were determined by HPLC using purified 5,4'-dihydroxy7-methoxy-6-methylflavan as a standard. Namely, MPE or a standard sample was dissolved in $\mathrm{MeOH}$ and quantified by HPLC using the following conditions. Develosil C30-UG 5 (\$4.6 mm×150 mm, Nomura Chemical Co. Ltd., Seto, Japan) and UV detector 210nm) were used. Flow late was fixed at $1.0 \mathrm{~mL} / \mathrm{min}$ and 60 (v/v) \% MeOH was used for solvent. Column temperature was set to $30^{\circ} \mathrm{C}$.

An allocation controller ordered test capsules according to the provided identification numbers using Statlight \#11 (Ver. 2.10, Yukms Inc.). Then, test capsules were allocated by class randomization to equalize the allocation ratio. Allocation was required in order for the means and standard deviation (SD) of MDQ scores and ages to not differ between groups. Information about allocation was strictly protected by third-party study allocation controllers not directly involved in the study, and this information was not disclosed to any other party until the subjects for analysis were determined at a clinical conference after study completion. 
Study protocol: This randomized, placebo-controlled, double-blind, parallel-group study was carried out at Takara Clinic (Medical Corporation Seishinkai, Tokyo, Japan), and statistical analysis was performed by ORTHOMEDICO Inc. The protocol was registered in the University Hospital Medical Information Network Clinical Trials Registry (UMIN0000334445). Subjects took two appropriate capsules (either MPE or placebo) daily after breakfast for 8 weeks. All subjects recorded a daily report, including capsule ingestion, body temperature, and menstruation, and answered a questionnaire.
The following items were examined at baseline, and at 4 and 8 weeks after intake. For primary outcomes, MDQ (Table 1) was used to assess the primary outcomes during, before, and after menstrual periods, and was completed before treatment, and after 4 and 8 weeks of intake. The answers to the questionnaire were categorized into 5 grades; 0: no experience of symptoms, 1: present (mild), 2: present (moderate), 3: present (strong), and 4: present (severe). All scores were treated to calculate upper scale scores in $\mathrm{MDQ}$.

Table 1. Factors included in MDQ

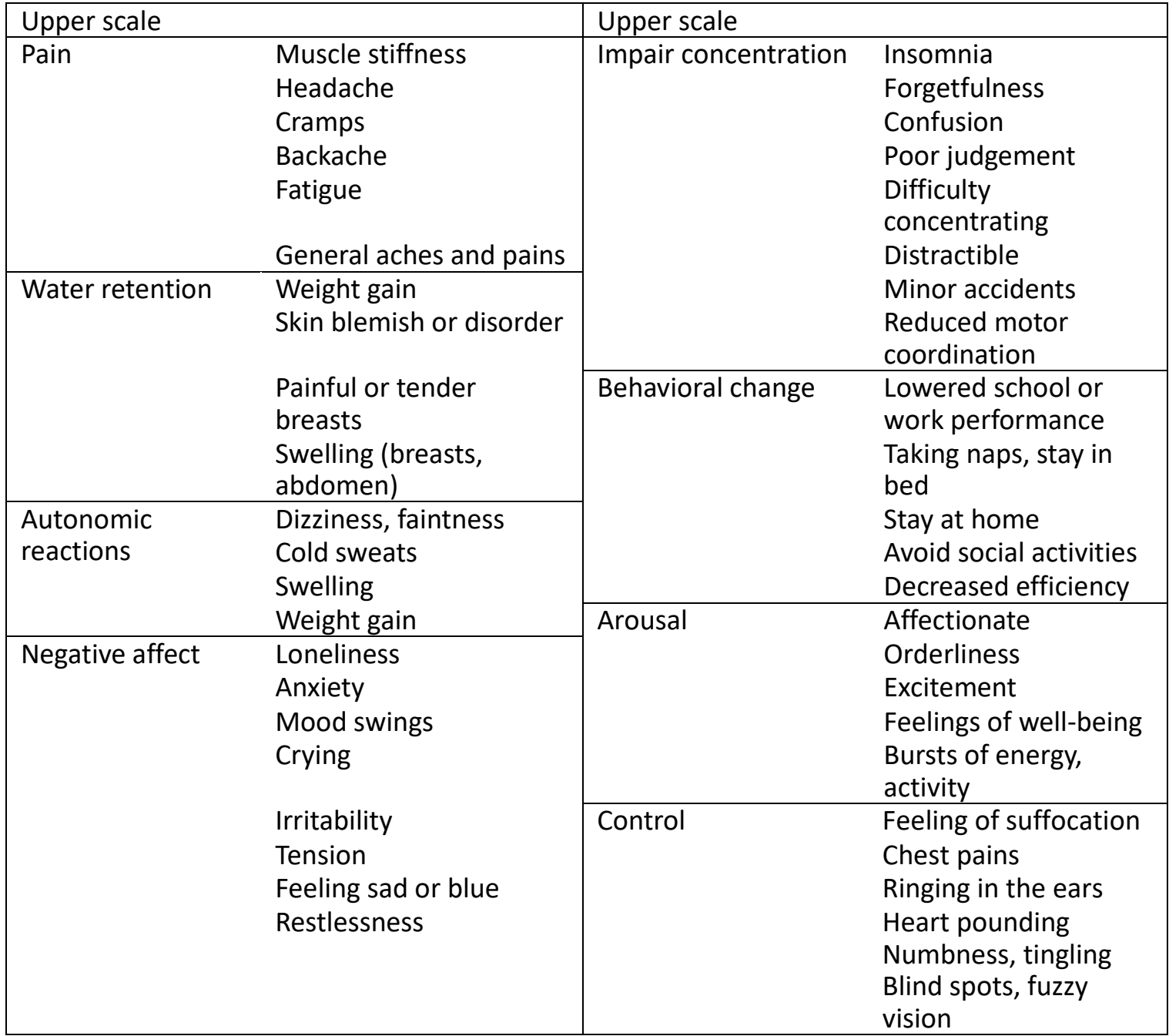


The SF-36 Health Survey was used to assess the secondary outcomes, and was completed before treatment, and after 4 and 8 weeks of intake. Answer sheets were processed according to the manual [21], and scores from 0 to 100 were calculated for the domains of physical functioning, role-physical, bodily pain, general health perception, vitality, social functioning, role-emotional, and mental health. Furthermore, these categories were divided into physical component summary (PCS), mental component summary (MCS), and role/social component summary (RCS), and calculated. A higher value indicates a better health condition.

Laboratory tests: Blood and urine samples were analyzed by LSI Medience Corporation (Tokyo, Japan). All items were examined at baseline, and at 4 and 8 weeks after intake. A venous blood sample was collected from an arm vein and the following tests were performed for safety assessment.

Hematology components were as follows: red blood cell count, leukocyte count, hemoglobin, hematocrit, platelet count, lymphocyte count, monocyte count, eosinophil count, and basophil count. Biochemical components were as follows: progesterone, estradiol, total protein, total bilirubin, urea nitrogen, creatinine, uric acid, total cholesterol, low-density lipoprotein (LDL) cholesterol, high-density lipoprotein (HDL) cholesterol, triglyceride, hemoglobin ( $\mathrm{Hb}$ ) A1c, blood glucose, amylase, creatine kinase (CK), aspartate aminotransferase (AST), alanine transaminase (ALT), $\mathrm{Y}$ glutamyltransferase ( $\gamma$-GTP), alkaline phosphatase $(\mathrm{ALP})$, lactate dehydrogenase (LDH), $\mathrm{Na}, \mathrm{K}, \mathrm{Cl}, \mathrm{Ca}, \mathrm{Fe}$, and leucine aminopeptidase (LAP).

In addition, urine samples were collected for qualitative evaluation, including protein, glucose, urobilinogen, bilirubin, ketone bodies, $\mathrm{pH}$, and occult blood.

Ethics, adherence, and compliance: This study was performed according to the Declaration of Helsinki (2013 revision) and was carried out in conformity with ethical considerations. This protocol was approved by the ethics committee of Takara Clinic (Medical Corporation Seishinkai, Tokyo, Japan) on September $11^{\text {th }}, 2018$ (Approved ID: 1807-1803-OZ02-03-TC), and substantial deviation from the protocol required authorization by the committee. All subjects received a full explanation of the protocol and purpose of the study before consenting to participate. No subject was part of the sponsoring or funding companies.

Statistical analysis: Estrogenic activity of 5,4'dihydroxy-7-methoxy-6-methylflavan was indicated mean and S.E. For statistical analysis, one-way analysis of variance (ANOVA) was performed, followed by Dunnett's test. The calculated MDQ and SF-36 scores are reported as the mean and SD. Two-way repeated measures ANCOVA or ANOVA followed by post hoc analysis was performed to detect significant differences between the two groups. Results of physical examination and blood tests were reported as the mean and SD. The Student's $t$-test was used to evaluate the significance of differences between before and after ingestion of the test sample. The $\chi^{2}$-test was used for urinalysis parameters, with normal and abnormal values being coded as " 1 " and " 0 ", respectively. We set the significance level at $5 \%$ with no adjustment for multiple comparisons. SPSS (Ver. 23.0, Japan IBM) or Microsoft Excel 2013 was used for statistical evaluation. 


\section{RESULTS}

Estrogenic acitivity of 5,4'-dihydroxy-7-methoxy-6- proliferaton assay, the compound promoted cell methylflavan: We identified 5,4'-dihydroxy-7- proliferation at 0.1 to $1 \mu \mathrm{g} / \mathrm{mL}$ (Figure 2B). Thus 5,4'methoxy-6-methylflavan as a candidate of dihydroxy-7-methoxy-6-methylflavan was found to be phytoestrogen (Figure $2 \mathrm{~A}$ ). As a result of MCF-7 phytoestrogen in MPE.

\section{A) 5,4'-dihydroxy-7-methoxy-} 6-methylflavan

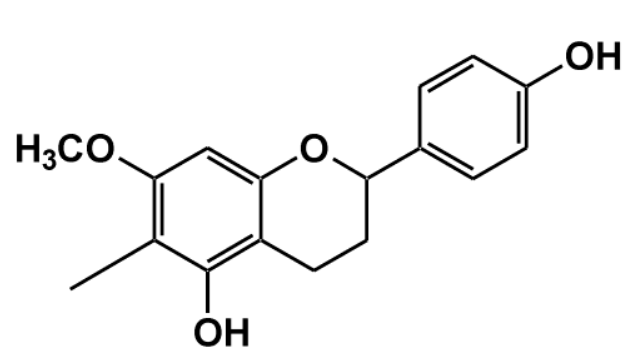

B) MCF-7 proliferation

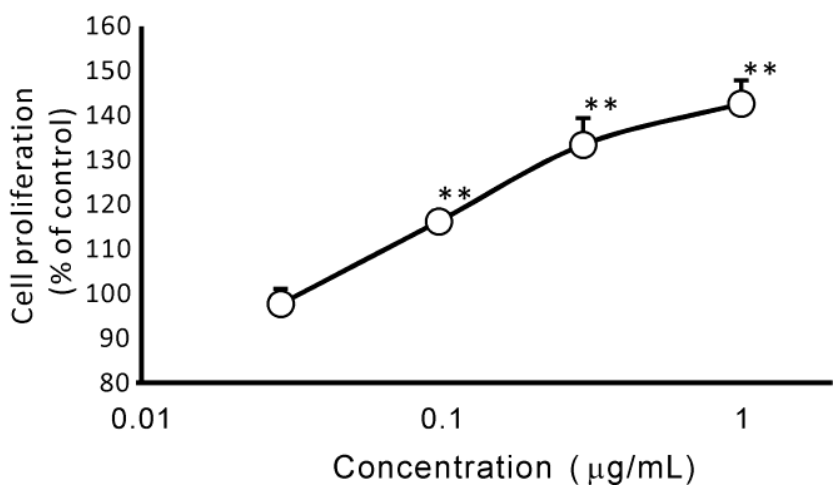

Figure 2. Estrogenic compound in MPE.

A) Chemical structure of 5,4'-dihydroxy-7-methoxy-6-methylflavan. B) Proliferative effect of 5,4'-dihydroxy-7-methoxy-6methylflavan in MCF-7. Each value represents mean with the S.E $(n=8)$. Asterisks denote significant differences from sample free group at $* *: p<0.01$.

Table 2. Profile of the participants.

\begin{tabular}{|c|c|c|c|c|}
\hline & \multicolumn{2}{|c|}{ Baseline } & \multicolumn{2}{|c|}{$8 w$} \\
\hline & MPE & Placebo & MPE & Placebo \\
\hline Age & $35.2 \pm 7.1$ & $34.7 \pm 5.9$ & & \\
\hline Height (cm) & $160.9 \pm 6.7$ & $160.2 \pm 5.2$ & & \\
\hline Body weight (kg) & $53.9 \pm 10.1$ & $52.0 \pm 5.3$ & $54.3 \pm 10.6$ & $52.6 \pm 6.2$ \\
\hline BMI $\left(\mathrm{kg} / \mathrm{m}^{2}\right)$ & $20.7 \pm 3.0$ & $20.3 \pm 1.7$ & $20.9 \pm 3.2$ & $20.5 \pm 2.0$ \\
\hline Body fat ratio (\%) & $23.0 \pm 5.5$ & $22.5 \pm 3.9$ & $22.8 \pm 5.9$ & $21.6 \pm 4.3$ \\
\hline $\begin{array}{l}\text { Systolic blood pressure } \\
(\mathrm{mmHg})\end{array}$ & $104.1 \pm 8.9$ & $106.5 \pm 11.3$ & $105.5 \pm 10.1$ & $106.7 \pm 8.8$ \\
\hline $\begin{array}{l}\text { Diastolic blood pressure } \\
(\mathrm{mmHg})\end{array}$ & $64.2 \pm 6.6$ & $65.3 \pm 8.6$ & $65.5 \pm 5.8$ & $66.4 \pm 8.7$ \\
\hline Pals rate (bpm) & $71.7 \pm 9.9$ & $69.8 \pm 9.4$ & $70.9 \pm 10.9$ & $69.3 \pm 9.5$ \\
\hline Body temperature $\left({ }^{\circ} \mathrm{C}\right)$ & $36.3 \pm 0.3$ & $36.4 \pm 0.4$ & $36.5 \pm 0.2^{*}$ & $36.4 \pm 0.3$ \\
\hline
\end{tabular}

Data is represented as the mean $\pm S D$ ( $n=21$ for MPE and $n=22$ for placebo). An asterisk denotes a significant difference from placebo at $p<0.05$. 
Study performance: The study was performed between October $1^{\text {st }}, 2018$ and December 28 ${ }^{\text {th }}, 2018$. During the study period, one subject in the MPE group was unable to track the study for personal reasons (Fig. 1), and was excluded from the analysis in the MPE group.
Accordingly, 21 subjects (35.2 \pm 7.1 years) were available for analysis in the MPE group, whereas 22 subjects (34.7 \pm 5.9 years) were available for the placebo group. The physical profile of the subjects included in analysis are shown in Table 2.

Table 3. Change of MDQ parameters during the menstrual period (most recent flow).

\begin{tabular}{|c|c|c|c|c|c|c|}
\hline & \multicolumn{2}{|c|}{ Baseline } & \multicolumn{2}{|c|}{$4 W$} & \multicolumn{2}{|c|}{$8 W$} \\
\hline & MPE & Placebo & MPE & Placebo & MPE & Placebo \\
\hline \multirow[t]{2}{*}{ Summary score } & $84.4 \pm 29.8$ & $83.4 \pm 27.1$ & $61.4 \pm 30.4$ & $61.5 \pm 25.3$ & $48.2 \pm 19.9 *$ & $64.7 \pm 27.1$ \\
\hline & & & $(-23.0 \pm 31.4)$ & $(-21.9 \pm 31.0)$ & $(-36.2 \pm 23.5)^{*}$ & $(-18.7 \pm 31.3)$ \\
\hline \multirow[t]{2}{*}{ Pain } & $14.6 \pm 3.5$ & $14.0 \pm 4.3$ & $11.4 \pm 4.7$ & $10.3 \pm 3.6$ & $9.7 \pm 5.5$ & $11.2 \pm 4.3$ \\
\hline & & & $(-3.2 \pm 4.6)$ & $(-3.7 \pm 5.3)$ & $(-5.0 \pm 4.8)$ & $(-2.8 \pm 4.8)$ \\
\hline \multirow[t]{2}{*}{ Water retention } & $9.2 \pm 3.4$ & $9.0 \pm 3.6$ & $6.9 \pm 3.3$ & $7.4 \pm 3.8$ & $5.0 \pm 3.1^{*}$ & $7.4 \pm 3.9$ \\
\hline & & & $(-2.3 \pm 2.9)$ & $(-1.6 \pm 3.5)$ & $(-4.2 \pm 2.9)^{*}$ & $(-1.6 \pm 4.0)$ \\
\hline \multicolumn{7}{|l|}{ Autonomic } \\
\hline reaction & $5.4 \pm 4.2$ & $5.7 \pm 3.4$ & $4.1 \pm 3.1$ & $3.7 \pm 2.8$ & $2.8 \pm 1.9$ & $3.8 \pm 2.9$ \\
\hline reaction & & & $(-8.8 \pm 27.9)$ & $(-14.2 \pm 23.0)$ & $(-2.6 \pm 3.7)$ & $(-1.9 \pm 3.5)$ \\
\hline \multirow[t]{2}{*}{ Negative affect } & $19.6 \pm 8.0$ & $19.6 \pm 6.8$ & $13.7 \pm 8.9$ & $13.8 \pm 7.0$ & $10.4 \pm 6.6$ & $13.6 \pm 5.7$ \\
\hline & & & $(-14.0 \pm 24.4)$ & $(-14.5 \pm 18.7)$ & $(-9.1 \pm 7.9)$ & $(-6.0 \pm 8.0)$ \\
\hline \multicolumn{7}{|l|}{ Impair } \\
\hline \multirow{2}{*}{ concentration } & $16.3 \pm 8.4$ & $16.2 \pm 8.7$ & $10.7 \pm 8.6$ & $12.4 \pm 7.3$ & $9.2 \pm 6.9 *$ & $12.8 \pm 6.6$ \\
\hline & & & $(-23.6 \pm 37.3)$ & $(-15.5 \pm 35.4)$ & $(-7.1 \pm 6.2)$ & $(-3.4 \pm 8.6)$ \\
\hline \multirow[t]{2}{*}{ Behavioral change } & $11.0 \pm 5.4$ & $10.8 \pm 4.8$ & $7.9 \pm 5.2$ & $6.7 \pm 4.1$ & $5.5 \pm 3.1$ & $7.5 \pm 5.5$ \\
\hline & & & $(-15.1 \pm 29.8)$ & $(-20.3 \pm 23.5)$ & $(-5.5 \pm 4.6)$ & $(-3.3 \pm 5.2)$ \\
\hline \multirow[t]{2}{*}{ Arousal } & $4.1 \pm 3.6$ & $4.9 \pm 3.8$ & $3.6 \pm 2.8$ & $5.0 \pm 3.3$ & $4.2 \pm 3.4$ & $5.3 \pm 3.4$ \\
\hline & & & $(-2.3 \pm 14.9)$ & $(0.4 \pm 21.0)$ & $(0.1 \pm 3.2)$ & $(0.5 \pm 3.7)$ \\
\hline \multirow[t]{2}{*}{ Control } & $4.2 \pm 4.5$ & $3.4 \pm 3.5$ & $3.0 \pm 4.1$ & $2.3 \pm 2.8$ & $1.3 \pm 2.5^{*}$ & $3.1 \pm 3.8$ \\
\hline & & & $(-8.2 \pm 33.4)$ & $(-7.0 \pm 21.0)$ & $(-2.9 \pm 5.0)^{*}$ & $(-0.2 \pm 3.6)$ \\
\hline
\end{tabular}

Actual scores and changes from baseline (in parenthesis) are represented as the mean and SD ( $n=21$ for MPE and $n=22$ for placebo). An asterisk indicates a significant difference from placebo at $p<0.05$. 
Table 4. Change in MDQ parameters in the premenstrual period (4 days before menstrual period).

\begin{tabular}{|c|c|c|c|c|c|c|}
\hline & \multicolumn{2}{|c|}{ Baseline } & \multicolumn{2}{|c|}{$4 W$} & \multicolumn{2}{|c|}{$8 W$} \\
\hline & MPE & Placebo & MPE & Placebo & MPE & Placebo \\
\hline \multirow[t]{2}{*}{ Summary score } & $79.7 \pm 24.2$ & $79.8 \pm 23.0$ & $54.8 \pm 28.3$ & $62.2 \pm 27.5$ & $41.8 \pm 28.3$ & $53.3 \pm 27.2$ \\
\hline & & & $(-25.0 \pm 31.7)$ & $(-17.6 \pm 33.0)$ & $(-37.6 \pm 32.8)$ & $(-26.5 \pm 34.9)$ \\
\hline \multirow[t]{2}{*}{ Pain } & $11.5 \pm 4.5$ & $11.6 \pm 4.7$ & $8.5 \pm 4.1$ & $10.1 \pm 4.3$ & $6.1 \pm 4.3$ & $8.7 \pm 4.8$ \\
\hline & & & $(-3.0 \pm 6.4)$ & $(-1.5 \pm 4.4)$ & $(-5.4 \pm 6.2)$ & $(-2.9 \pm 6.3)$ \\
\hline \multirow[t]{2}{*}{ Water retention } & $8.6 \pm 3.2$ & $9.7 \pm 4.0$ & $6.9 \pm 3.6$ & $7.0 \pm 4.3$ & $5.0 \pm 3.9$ & $6.8 \pm 4.4$ \\
\hline & & & $(-1.7 \pm 3.5)$ & $(-2.6 \pm 3.8)$ & $(-3.6 \pm 5.1)$ & $(-2.9 \pm 4.9)$ \\
\hline \multirow[t]{2}{*}{ Autonomic reaction } & $4.8 \pm 4.2$ & $4.5 \pm 3.1$ & $3.6 \pm 2.7$ & $3.1 \pm 2.2$ & $2.5 \pm 2.4$ & $2.6 \pm 2.1$ \\
\hline & & & $(-1.2 \pm 4.5)$ & $(-1.5 \pm 3.2)$ & $(-2.3 \pm 4.6)$ & $(-1.9 \pm 3.4)$ \\
\hline \multirow[t]{2}{*}{ Negative affect } & $20.5 \pm 6.6$ & $20.7 \pm 6.2$ & $13.4 \pm 8.7$ & $14.2 \pm 7.1$ & $9.9 \pm 8.4$ & $11.9 \pm 6.5$ \\
\hline & & & $(-7.0 \pm 9.4)$ & $(-6.5 \pm 7.6)$ & $(-10.6 \pm 9.3)$ & $(-8.8 \pm 8.7)$ \\
\hline \multicolumn{7}{|l|}{ Impair } \\
\hline \multirow{2}{*}{ concentration } & $15.5 \pm 7.6$ & $15.6 \pm 7.4$ & $9.7 \pm 8.0$ & $12.8 \pm 7.7$ & $7.6 \pm 7.6$ & $10.6 \pm 6.1$ \\
\hline & & & $(-5.8 \pm 8.0)$ & $(-2.8 \pm 9.5)$ & $(-8.0 \pm 7.9)$ & $(-5.0 \pm 8.4)$ \\
\hline \multirow[t]{2}{*}{ Behavioral change } & $10.5 \pm 5.0$ & $9.8 \pm 4.4$ & $6.7 \pm 4.6$ & $6.9 \pm 4.4$ & $4.4 \pm 4.5$ & $5.0 \pm 3.8$ \\
\hline & & & $(-3.8 \pm 6.3)$ & $(-2.9 \pm 5.5)$ & $(-6.1 \pm 5.9)$ & $(-4.8 \pm 5.7)$ \\
\hline \multirow[t]{2}{*}{ Arousal } & $4.5 \pm 4.0$ & $4.6 \pm 3.7$ & $3.4 \pm 3.0^{*}$ & $5.4 \pm 3.6$ & $5.4 \pm 3.4$ & $5.0 \pm 3.4$ \\
\hline & & & $(-1.1 \pm 3.4)^{*}$ & $(0.7 \pm 4.7)$ & $(0.9 \pm 4.1)$ & $(0.4 \pm 4.3)$ \\
\hline \multirow[t]{2}{*}{ Control } & $3.9 \pm 3.6$ & $3.3 \pm 3.1$ & $2.6 \pm 3.5$ & $2.7 \pm 2.7$ & $1.0 \pm 2.4 *$ & $2.8 \pm 3.6$ \\
\hline & & & $(-1.3 \pm 4.6)$ & $(-0.6 \pm 3.2)$ & $(-2.9 \pm 4.2)$ & $(-0.5 \pm 3.4)$ \\
\hline
\end{tabular}

Actual scores and changes from baseline (in parenthesis) are represented as the mean and SD ( $n=21$ for MPE and $n=22$ for placebo). An asterisk indicates a significant difference from placebo at $*: p<0.05$.

MDQ parameters: The MDQ parameters during, 4 days before, and in the postmenstrual period are presented in Tables 3-5. After 8-week intervention, the summary score, water retention, impaired concentration, and control were significantly lower in the MPE group than in the placebo group $(p<0.05)$ during the menstrual period (Table 3). On the other hand, control in pre(Table 4) and postmenstrual (Table 5) periods in the MPE group after the 8-week intervention was significantly lower than that in the placebo group ( $p<$ 0.05). Moreover, arousal in the premenstrual period after 4-week intervention in the MPE group was significantly lower than in the placebo group (Table 4). 
Table 5. Change in MDQ parameters in the postmenstrual period (remainder of cycle).

\begin{tabular}{|c|c|c|c|c|c|c|}
\hline & \multicolumn{2}{|c|}{ Baseline } & \multicolumn{2}{|c|}{$4 \mathrm{~W}$} & \multicolumn{2}{|c|}{$8 W$} \\
\hline & MPE & Placebo & MPE & Placebo & MPE & Placebo \\
\hline \multirow[t]{2}{*}{ Summary score } & $23.5 \pm 14.3$ & $22.6 \pm 14.6$ & $21.8 \pm 12.8$ & $28.1 \pm 19.4$ & $20.0 \pm 13.2$ & $28.5 \pm 27.2$ \\
\hline & & & $(-1.7 \pm 12.6)$ & $(5.5 \pm 17.5)$ & $(-3.4 \pm 14.9)$ & $(6.0 \pm 19.7)$ \\
\hline \multirow[t]{2}{*}{ Pain } & $2.9 \pm 2.3$ & $2.9 \pm 3.0$ & $3.3 \pm 3.1$ & $3.8 \pm 3.1$ & $2.5 \pm 2.5$ & $3.5 \pm 2.9$ \\
\hline & & & $(0.4 \pm 3.0)$ & $(0.9 \pm 2.1)$ & $(-0.4 \pm 2.7)$ & $(0.6 \pm 3.6)$ \\
\hline \multirow[t]{2}{*}{ Water retention } & $1.7 \pm 1.5$ & $1.6 \pm 2.0$ & $1.7 \pm 1.3$ & $2.1 \pm 2.0$ & $1.4 \pm 1.6$ & $2.0 \pm 2.4$ \\
\hline & & & $(0.0 \pm 1.7)$ & $(0.5 \pm 1.7)$ & $(-0.2 \pm 1.7)$ & $(0.5 \pm 2.3)$ \\
\hline \multirow{3}{*}{$\begin{array}{l}\text { Autonomic } \\
\text { reaction }\end{array}$} & & & & & & \\
\hline & $1.0 \pm 1.2$ & $0.8 \pm 1.6$ & & & & \\
\hline & & & $(-0.4 \pm 1.3)$ & $(-0.1 \pm 2.1)$ & $(-0.2 \pm 1.3)$ & $(0.5 \pm 1.4)$ \\
\hline \multirow[t]{2}{*}{ Negative affect } & $4.4 \pm 6.3$ & $4.5 \pm 4.7$ & $5.0 \pm 5.6$ & $5.9 \pm 6.2$ & $4.0 \pm 4.5$ & $5.9 \pm 5.6$ \\
\hline & & & $(0.6 \pm 6.9)$ & $(1.3 \pm 4.5)$ & $(-0.3 \pm 7.3)$ & $(1.3 \pm 6.0)$ \\
\hline \multicolumn{7}{|l|}{ Impair } \\
\hline concentration & $3.5 \pm 3.5$ & $3.7 \pm 4.2$ & & & & \\
\hline & & & $(-0.5 \pm 2.4)$ & $(1.3 \pm 4.5)$ & $(-0.7 \pm 3.9)$ & $(1.3 \pm 4.3)$ \\
\hline \multirow[t]{2}{*}{ Behavioral change } & $1.9 \pm 2.4$ & $1.1 \pm 1.5$ & $1.1 \pm 1.3$ & $2.1 \pm 3.6$ & $1.3 \pm 1.9$ & $2.0 \pm 3.0$ \\
\hline & & & $(-0.8 \pm 2.6)$ & $(1.0 \pm 3.2)$ & $(-0.6 \pm 2.9)$ & $(0.9 \pm 2.6)$ \\
\hline \multirow[t]{2}{*}{ Arousal } & $7.2 \pm 3.6$ & $6.9 \pm 4.4$ & $6.2 \pm 3.7$ & $7.0 \pm 4.5$ & $6.8 \pm 4.3$ & $7.2 \pm 5.0$ \\
\hline & & & $(-1.0 \pm 4.9)$ & $(0.1 \pm 4.6)$ & $(-0.4 \pm 4.9)$ & $(0.3 \pm 4.6)$ \\
\hline \multirow[t]{2}{*}{ Control } & $0.8 \pm 1.3$ & $1.0 \pm 1.8$ & $0.7 \pm 0.8$ & $1.4 \pm 2.2$ & $0.3 \pm 0.5^{*}$ & $1.7 \pm 2.8$ \\
\hline & & & $(-0.1 \pm 1.5)$ & $(0.4 \pm 1.7)$ & $(-0.5 \pm 1.2)$ & $(0.7 \pm 1.9)$ \\
\hline
\end{tabular}

Actual scores and changes from baseline (in parenthesis) are represented as the mean and SD ( $n=21$ for MPE and $n=22$ for placebo). An asterisk indicates a significant difference from placebo at $*$ : $p<0.05$.

SF-36 parameters: The outcome of SF-36 is presented in Table 6. After 4 weeks, the change in RCS was significantly lower in the MPE group than in the placebo group $(p<0.05)$. The reduction reflects deteriorating effects of MPE in subjects when they performed their role at work or when completed house chores. However, this reduction was improved by the 8-week period. The other parameters in the MPE group did not significantly change after 4 or 8 weeks of MPE ingestion. 
Table 6. Change in SF-36 parameters

\begin{tabular}{|c|c|c|c|c|c|c|}
\hline & \multicolumn{2}{|c|}{ Baseline } & \multicolumn{2}{|c|}{$4 W$} & \multicolumn{2}{|c|}{$8 W$} \\
\hline & MPE & Placebo & MPE & Placebo & MPE & Placebo \\
\hline PCS & $52.4 \pm 7.8$ & $57.0 \pm 9.5$ & $\begin{array}{l}54.9 \pm 6.9 \\
(2.4 \pm 6.3)\end{array}$ & $\begin{array}{l}57.8 \pm 8.4 \\
(0.8 \pm 7.3)\end{array}$ & $\begin{array}{l}55.3 \pm 5.8 \\
(2.9 \pm 6.7)\end{array}$ & $\begin{array}{c}56.9 \pm 5.8 \\
(-0.1 \pm 8.7)\end{array}$ \\
\hline MCS & $48.2 \pm 8.5$ & $45.0 \pm 7.4$ & $\begin{array}{l}49.3 \pm 9.3 \\
(1.1 \pm 8.6)\end{array}$ & $\begin{array}{c}43.8 \pm 7.2 \\
(-1.2 \pm 6.2)\end{array}$ & $\begin{array}{l}50.1 \pm 6.4 \\
(1.9 \pm 8.6)\end{array}$ & $\begin{array}{l}47.5 \pm 5.3 \\
(2.6 \pm 6.9)\end{array}$ \\
\hline RCS & $44.1 \pm 12.1$ & $37.7 \pm 11.9$ & $\begin{array}{c}43.1 \pm 11.2 \\
\left(-1.0 \pm 12.4^{*}\right)\end{array}$ & $\begin{array}{c}44.5 \pm 11.2 \\
(6.7 \pm 9.9)\end{array}$ & $\begin{array}{l}46.9 \pm 8.9 \\
(2.9 \pm 9.5)\end{array}$ & $\begin{array}{l}40.7 \pm 13.2 \\
(2.9 \pm 11.5)\end{array}$ \\
\hline $\begin{array}{l}\text { Physical } \\
\text { functioning }\end{array}$ & $53.2 \pm 9.2$ & $53.4 \pm 9.5$ & $\begin{array}{l}54.2 \pm 5.9 \\
(1.0 \pm 5.0)\end{array}$ & $\begin{array}{l}55.2 \pm 2.3 \\
(1.8 \pm 9.0)\end{array}$ & $\begin{array}{l}56.0 \pm 3.2 \\
(2.8 \pm 8.1)\end{array}$ & $\begin{array}{l}54.6 \pm 4.0 \\
(1.1 \pm 9.8)\end{array}$ \\
\hline $\begin{array}{l}\text { Physical role } \\
\text { functioning }\end{array}$ & $46.1 \pm 12.9$ & $43.3 \pm 12.4$ & $\begin{array}{l}46.7 \pm 10.6 \\
(0.6 \pm 11.9)\end{array}$ & $\begin{array}{c}48.3 \pm 8.6 \\
(5.0 \pm 12.7)\end{array}$ & $\begin{array}{c}48.0 \pm 9.4 \\
(1.9 \pm 12.1)\end{array}$ & $\begin{array}{l}44.4 \pm 11.7 \\
(1.1 \pm 13.2)\end{array}$ \\
\hline Bodily pain & $42.3 \pm 11.5$ & $46.8 \pm 9.1$ & $\begin{array}{l}46.7 \pm 12.7 \\
(4.4 \pm 13.5)\end{array}$ & $\begin{array}{l}50.7 \pm 8.4 \\
(4.0 \pm 8.3)\end{array}$ & $\begin{array}{l}49.6 \pm 11.1 \\
(7.3 \pm 12.4)\end{array}$ & $\begin{array}{l}51.0 \pm 7.9 \\
(4.3 \pm 8.7)\end{array}$ \\
\hline $\begin{array}{l}\text { General health } \\
\text { perception }\end{array}$ & $53.0 \pm 10.3$ & $50.9 \pm 8.9$ & $\begin{array}{l}54.7 \pm 9.4 \\
(1.1 \pm 8.2)\end{array}$ & $\begin{array}{l}50.4 \pm 8.4 \\
(-0.5 \pm 5.7)\end{array}$ & $\begin{array}{l}54.9 \pm 7.1 \\
(1.9 \pm 7.7)\end{array}$ & $\begin{array}{l}51.8 \pm 7.3 \\
(0.9 \pm 5.9)\end{array}$ \\
\hline Vitality & $47.2 \pm 9.3$ & $42.4 \pm 7.8$ & $\begin{array}{l}47.1 \pm 9.8 \\
(-0.2 \pm 8.5)\end{array}$ & $\begin{array}{l}42.1 \pm 7.5 \\
(-0.3 \pm 7.5)\end{array}$ & $\begin{array}{l}48.6 \pm 6.5 \\
(1.4 \pm 9.8)\end{array}$ & $\begin{array}{l}44.3 \pm 8.3 \\
(1.9 \pm 9.9)\end{array}$ \\
\hline $\begin{array}{l}\text { Social role } \\
\text { functioning }\end{array}$ & $46.3 \pm 11.9$ & $40.0 \pm 9.2$ & $\begin{array}{c}46.9 \pm 9.5 \\
(0.6 \pm 12.4)\end{array}$ & $\begin{array}{l}46.2 \pm 9.4 \\
(6.1 \pm 9.4)\end{array}$ & $\begin{array}{c}50.9 \pm 8.0 \\
(4.6 \pm 10.6)\end{array}$ & $\begin{array}{l}46.5 \pm 11.0 \\
(6.4 \pm 9.9)\end{array}$ \\
\hline $\begin{array}{l}\text { Emotional role } \\
\text { functioning }\end{array}$ & $45.8 \pm 10.7$ & $42.1 \pm 9.8$ & $\begin{array}{l}45.6 \pm 10.1 \\
(-0.2 \pm 9.4)\end{array}$ & $\begin{array}{l}46.0 \pm 10.8 \\
(4.0 \pm 9.4)\end{array}$ & $\begin{array}{l}49.9 \pm 7.9 \\
(4.2 \pm 8.9)\end{array}$ & $\begin{array}{r}43.4 \pm 11.2 \\
(1.3 \pm 9.6)\end{array}$ \\
\hline Mental health & $47.2 \pm 10.7$ & $43.3 \pm 7.3$ & $\begin{array}{l}48.2 \pm 8.2 \\
(1.0 \pm 9.5)\end{array}$ & $\begin{array}{l}44.4 \pm 9.1 \\
(1.1 \pm 7.5)\end{array}$ & $\begin{array}{l}49.4 \pm 7.2 \\
(2.2 \pm 9.5)\end{array}$ & $\begin{array}{l}45.6 \pm 7.4 \\
(2.3 \pm 6.5)\end{array}$ \\
\hline
\end{tabular}

The scores and changes from baseline (in parenthesis) are represented as the mean and SD ( $n=21$ for MPE and $n=22$ for placebo). An asterisk indicates a significant difference from placebo at $*$ : $p<0.05$. 
Laboratory data and adverse effects: The blood pressure, pulse rate, and body temperature are listed in Table 2. The body temperature in the MPE group was slightly higher than the placebo group's temperature after the 8-week intervention. Blood progesterone and estradiol, and hematology parameters are shown in Table 7. No significant changes were observed between the two groups. Biochemical parameters are shown in
Tables 8. Before intervention, significant differences were observed in hemoglobin, $\mathrm{HbA1c}$, and Fe between the different groups. After the 8-week intervention, only total bilirubin was higher than the control. However, these changes were all within reference ranges. Urinalysis parameters did not change in either group (Table 9).

Table 7. Changes in the prostaglandin, estradiol, and hematology parameters

\begin{tabular}{|c|c|c|c|c|c|c|c|}
\hline & \multicolumn{2}{|c|}{ Baseline } & \multicolumn{2}{|c|}{$\begin{array}{c}\text { After } 4 \text { weeks of } \\
\text { ingestion }\end{array}$} & \multicolumn{2}{|c|}{ After 8 weeks of ingestion } & \multirow[t]{2}{*}{$\begin{array}{c}\text { Standard } \\
\text { value }\end{array}$} \\
\hline & MPE & Placebo & MPE & Placebo & MPE & Placebo & \\
\hline Progesterone $(\mathrm{ng} / \mathrm{mL})$ & $3.3 \pm 5.4$ & $1.1 \pm 3.3$ & $2.4 \pm 5.8$ & $2.7 \pm 5.3$ & $2.8 \pm 4.2$ & $2.1 \pm 4.6$ & \\
\hline Estradiol (pg/mL) & $133 \pm 131$ & $167 \pm 126$ & $121 \pm 99$ & $145 \pm 104$ & $136 \pm 87$ & $118 \pm 103$ & \\
\hline Red blood cells $\left(\times 10^{4}\right.$ cells $\left./ \mu \mathrm{L}\right)$ & $420 \pm 40$ & $431 \pm 35$ & $424 \pm 36$ & $436 \pm 30$ & $426 \pm 31$ & $438 \pm 31$ & $380-500$ \\
\hline Leukocytes (cells/ $\mu \mathrm{L}$ ) & $5419 \pm 1333$ & $5418 \pm 1191$ & $5819 \pm 1592$ & $5359 \pm 1748$ & $5828 \pm 1658$ & $5036 \pm 1338$ & $3300-9000$ \\
\hline Hemoglobin (g/dL) & $12.4 \pm 1.1^{*}$ & $13.1 \pm 0.9$ & $12.5 \pm 1.1$ & $13.2 \pm 1.0$ & $12.6 \pm 1.0$ & $13.2 \pm 0.9$ & $11.5-15.0$ \\
\hline Hematocrit (\%) & $39.5 \pm 3.2$ & $41.2 \pm 2.9$ & $39.9 \pm 2.9$ & $42.0 \pm 2.6$ & $40.4 \pm 2.6$ & $42.3 \pm 2.8$ & $34.8-45.0$ \\
\hline Platelets $\left(\times 10^{4}\right.$ cells $\left./ \mu \mathrm{L}\right)$ & $26.5 \pm 4.7$ & $28.2 \pm 4.7$ & $27.4 \pm 5.0$ & $29.5 \pm 4.2$ & $27.6 \pm 4.7$ & $29.6 \pm 4.4$ & $14.0-34.0$ \\
\hline Neutrophils (cells/ $\mu \mathrm{L}$ ) & $3275 \pm 1128$ & $3157 \pm 931$ & $3654 \pm 1354$ & $3268 \pm 1506$ & $3618 \pm 1223$ & $2914 \pm 1199$ & \\
\hline Lymphocytes (cells/ $\mu \mathrm{L}$ ) & $1753 \pm 428$ & $1792 \pm 595$ & $1753 \pm 576$ & $1625 \pm 483$ & $1775 \pm 565$ & $1692 \pm 470$ & \\
\hline Monocytes (cells/ $\mu \mathrm{L}$ ) & $251 \pm 72$ & $264 \pm 69$ & $264 \pm 100$ & $285 \pm 106$ & $285 \pm 100$ & $251 \pm 56$ & \\
\hline Eosinophils (cells/ $\mu \mathrm{L}$ ) & $108 \pm 103$ & $165 \pm 138$ & $118 \pm 120$ & $144 \pm 137$ & $118 \pm 118$ & $145 \pm 97$ & \\
\hline Basophils (cells/ $\mu \mathrm{L}$ ) & $29.8 \pm 13.7$ & $37.8 \pm 25.6$ & $28.0 \pm 16.4$ & $36.2 \pm 25.5$ & $31.1 \pm 17.0$ & $32.6 \pm 18.7$ & \\
\hline
\end{tabular}

Actual scores and changed scores (in parenthesis) are presented as the mean and SD ( $n=21$ for MPE and $n=22$ for placebo). An asterisk indicates a significant difference from placebo at *: $p<0.05$. 
Table 8. Changes in biochemical parameters

\begin{tabular}{|c|c|c|c|c|c|c|c|}
\hline & \multicolumn{2}{|c|}{ Baseline } & \multicolumn{2}{|c|}{$\begin{array}{l}\text { After } 4 \text { weeks of } \\
\text { ingestion }\end{array}$} & \multicolumn{2}{|c|}{ After 8 weeks of ingestion } & \multirow[t]{2}{*}{ Standard value } \\
\hline & MPE & Placebo & MPE & Placebo & MPE & Placebo & \\
\hline Total protein (g/dL) & $7.0 \pm 0.4$ & $7.0 \pm 0.3$ & $6.9 \pm 0.4$ & $6.9 \pm 0.3$ & $6.9 \pm 0.5$ & $6.9 \pm 0.4$ & $6.7-8.3$ \\
\hline Total bilirubin (mg/dL) & $0.72 \pm 0.26$ & $0.71 \pm 0.21$ & $0.76 \pm 0.22$ & $0.74 \pm 0.16$ & $0.77 \pm 0.15^{*}$ & $0.68 \pm 0.17$ & $0.2-1.2$ \\
\hline Urea N (mg/dL) & $11.0 \pm 2.6$ & $11.3 \pm 3.0$ & $11.9 \pm 3.5$ & $12.2 \pm 3.5$ & $11.2 \pm 3.1$ & $12.0 \pm 2.8$ & \\
\hline Creatinine (mg/dL) & $0.63 \pm 0.10$ & $0.62 \pm 0.09$ & $0.63 \pm 0.10$ & $0.61 \pm 0.08$ & $0.63 \pm 0.12$ & $0.59 \pm 0.08$ & $0.47-0.79$ \\
\hline Uric acid (mg/dL) & $4.1 \pm 0.9$ & $4.3 \pm 1.0$ & $4.3 \pm 1.0$ & $4.1 \pm 0.9$ & $4.1 \pm 0.7$ & $4.1 \pm 0.9$ & $2.5-7.0$ \\
\hline Total cholesterol (mg/dL) & $203 \pm 39$ & $191 \pm 28$ & $207 \pm 42$ & $202 \pm 30$ & $213 \pm 36$ & $198 \pm 30$ & $120-219$ \\
\hline LDL cholesterol (mg/dL) & $112 \pm 38$ & $107 \pm 27$ & $112 \pm 39$ & $110 \pm 28$ & $116 \pm 35$ & $108 \pm 29$ & $65-139$ \\
\hline HDL cholesterol (mg/dL) & $77 \pm 16$ & $72 \pm 15$ & $83 \pm 20$ & $78 \pm 14$ & $84 \pm 20$ & $75 \pm 13$ & $40-95$ \\
\hline Triglyceride (mg/dL) & $68 \pm 22$ & $61 \pm 32$ & $59 \pm 24$ & $60 \pm 24$ & $60 \pm 26$ & $64 \pm 30$ & $30-149$ \\
\hline HbA1c (\%) & $5.3 \pm 0.2 *$ & $5.1 \pm 0.2$ & $5.2 \pm 0.2$ & $5.1 \pm 0.3$ & $5.2 \pm 0.2$ & $5.1 \pm 0.3$ & $4.6-6.2$ \\
\hline Blood glucose (mg/dL) & $82 \pm 5$ & $79 \pm 10$ & $82 \pm 4$ & $79 \pm 8$ & $82 \pm 5$ & $80 \pm 7$ & 70-109 \\
\hline Amylase (U/L) & $79 \pm 26$ & $72 \pm 20$ & $79 \pm 30$ & $76 \pm 25$ & $77 \pm 29$ & $79 \pm 24$ & $40-122$ \\
\hline CK (U/L) & $91 \pm 41$ & $85 \pm 50$ & $155 \pm 251$ & $88 \pm 46$ & $104 \pm 66$ & $84 \pm 35$ & $40-150$ \\
\hline AST (U/L) & $18.1 \pm 5.5$ & $18.6 \pm 4.6$ & $20.5 \pm 10.4$ & $19.0 \pm 3.6$ & $19.5 \pm 5.7$ & $19.1 \pm 5.3$ & $10-40$ \\
\hline $\operatorname{ALT}(\mathrm{U} / \mathrm{L})$ & $12.2 \pm 9.1$ & $13.7 \pm 6.4$ & $13.4 \pm 8.2$ & $14.2 \pm 5.2$ & $13.9 \pm 8.8$ & $14.5 \pm 6.3$ & $5-45$ \\
\hline v-GTP (U/L) & $15.4 \pm 3.9$ & $16.9 \pm 5.0$ & $15.4 \pm 4.4$ & $17.5 \pm 6.3$ & $16.3 \pm 6.0$ & $17.6 \pm 6.4$ & $<30$ \\
\hline $\operatorname{ALP}(U / L)$ & $154 \pm 42$ & $144 \pm 36$ & $165 \pm 49$ & $149 \pm 32$ & $159 \pm 41$ & $154 \pm 37$ & $100-325$ \\
\hline $\mathrm{LDH}(\mathrm{U} / \mathrm{L})$ & $175 \pm 29$ & $176 \pm 30$ & $177 \pm 35$ & $173 \pm 40$ & $175 \pm 29$ & $177 \pm 38$ & $120-240$ \\
\hline $\mathrm{Na}(\mathrm{mEq} / \mathrm{L})$ & $140 \pm 2$ & $140 \pm 2$ & $139 \pm 1$ & $139 \pm 1$ & $139 \pm 1$ & $139 \pm 1$ & $137-147$ \\
\hline $\mathrm{K}(\mathrm{mEq} / \mathrm{L})$ & $3.9 \pm 0.3$ & $3.9 \pm 0.3$ & $3.8 \pm 0.3$ & $3.9 \pm 0.3$ & $3.9 \pm 0.3$ & $4.1 \pm 0.4$ & $3.5-5.0$ \\
\hline $\mathrm{Cl}(\mathrm{mEq} / \mathrm{L})$ & $102 \pm 1$ & $101 \pm 1$ & $101 \pm 2$ & $101 \pm 1$ & $101 \pm 1$ & $101 \pm 1$ & $98-108$ \\
\hline $\mathrm{Ca}(\mathrm{mg} / \mathrm{dL})$ & $8.9 \pm 0.3$ & $9.0 \pm 0.3$ & $9.0 \pm 0.3$ & $9.0 \pm 0.2$ & $9.1 \pm 0.3$ & $9.1 \pm 0.3$ & $8.4-10.4$ \\
\hline $\mathrm{Fe}(\mu \mathrm{g} / \mathrm{dL})$ & $66 \pm 28 *$ & $91 \pm 40$ & $72 \pm 33$ & $86 \pm 32$ & $82 \pm 39$ & $90 \pm 35$ & $40-180$ \\
\hline LAP (U/L) & $45 \pm 4$ & $47 \pm 4$ & $44 \pm 5$ & $47 \pm 5$ & $44 \pm 6$ & $46 \pm 4$ & $37-61$ \\
\hline
\end{tabular}

Actual scores and changed scores (in parenthesis) are represented as the mean and SD ( $n=21$ for MPE and $n=22$ for placebo). An asterisk indicates a significant difference from placebo at *: $p<0.05$. 
Table 9. Changes in urine parameters

\begin{tabular}{|c|c|c|c|c|}
\hline & Week & MPE & Placebo & Standard value \\
\hline \multirow[t]{3}{*}{ Protein } & 0 & (nor):20, (ab):1 & (nor):22, (ab):0 & (nor) \\
\hline & 4 & (nor):18, (ab):3 & (nor):21, (ab):1 & \\
\hline & 8 & (nor):19, (ab):2 & (nor):21, (ab):1 & \\
\hline \multirow[t]{3}{*}{ Glucose } & 0 & $($ nor): $21,(a b): 0$ & (nor):22, (ab):0 & (nor) \\
\hline & 4 & (nor):21, (ab):0 & (nor):22, (ab):0 & \\
\hline & 8 & $(n o r): 21,(a b): 0$ & (nor):22, (ab):0 & \\
\hline \multirow[t]{3}{*}{ Urobilinogen } & 0 & (nor):20, (ab):1 & (nor):22, (ab):0 & (nor) \\
\hline & 4 & (nor):21, (ab):0 & (nor):22, (ab):0 & \\
\hline & 8 & $(n o r): 21,(a b): 0$ & (nor):22, (ab):0 & \\
\hline \multirow[t]{3}{*}{ Bilirubin } & 0 & (nor):21, (ab):0 & (nor):22, (ab):0 & (nor) \\
\hline & 4 & $(n o r): 21,(a b): 0$ & (nor):22, (ab):0 & \\
\hline & 8 & $($ nor): $21,(a b): 0$ & (nor):22, (ab):0 & \\
\hline \multirow[t]{3}{*}{$\mathrm{pH}$} & 0 & (nor):20, (ab):1 & $($ nor):21, $(a b): 1$ & (nor) \\
\hline & 4 & (nor):21, (ab):0 & (nor):21, (ab):1 & \\
\hline & 8 & (nor):20, (ab):1 & (nor):19, (ab):3 & \\
\hline \multirow[t]{3}{*}{ Occult blood } & 0 & (nor):20, (ab):1 & (nor):19, (ab):3 & (nor) \\
\hline & 4 & (nor):16, (ab):5 & $($ nor):20, (ab):2 & \\
\hline & 8 & (nor):19, (ab):2 & (nor):19, (ab):3 & \\
\hline Ketone & 0 & (nor):20, (ab):1 & (nor):20, (ab):2 & (nor) \\
\hline \multirow[t]{2}{*}{ bodies } & 4 & (nor):20, (ab):1 & $($ nor):20, $(a b): 2$ & \\
\hline & 8 & (nor):21, (ab):0 & (nor):21, (ab):1 & \\
\hline
\end{tabular}

Data is presented as number of subjects with normal values (nor) or abnormal values (ab).

This study evaluated the effects of daily MPE (100 mg) ingestion on indefinite complaints by Japanese women during the pre- and postmenstrual period. As the primary outcome, MDQ during the menstrual period, parameters related to water retention, arousal, concentration, and control were improved by MPE (Table 3). Regarding each question in MDQ, 8-week ingestion of MPE significantly improved "cold sweats [MPE $0.0(0.0-0.0)$ vs Placebo $0.0(0.0-1.0), p<0.05]$ ]" and “crying [MPE 0.0 (0.0-1.0) vs Placebo 1.0 (0.0-2.0), $p<0.05]$ " during the menstrual period, and "headache [MPE 1.0 (0.0-1.0) vs Placebo 2.0 (1.0-2.8), $p<0.05], "$ "reduced motor coordination [MPE 0.0 (0.0-1.0) vs Placebo 1.0 (0.3-2.0), $p<0.01]$," and "feelings of suffocation [MPE 0.0 (0.0-0.0) vs Placebo 0.0 (0.0-1.0), $p<0.05]^{\prime \prime}$ in the premenstrual period. Moreover, MPE ameliorated the "feelings of suffocation" in the postmenstrual period (MPE 0.0 (0.0-0.0) vs Placebo 0.0 
(0.0-1.0), $p<0.05)$. However, MPE did not provide pain relief; except for headache, and is unlikely to affect autonomic nerve disorder or mental depression because it did not improve "autonomic reactions," "negative affect," or "impaired concentration." MPE may improve water retention, mood, poor concentration, and discomfort of the upper body, which belong to "control." By the 4-week intervention of MPE (100 mg), scores of arousal in the premenstrual period decreased compared with those of the placebo group (Table 3). However, this effect disappeared by the $8^{\text {th }}$ week. This phenomenon may be caused by the decreasing MDQ value in the placebo group by placebo effect in addition to the decrease in effects of MPE.

Premenstrual syndromes (PMS) include physical and mental anxieties, premenstrual dysphoric disorder, and dysmenorrhea [22]. Symptoms in PMS last until the menstrual period, and the combination of low-dose estrogen and progesterone is effective against physical and mental symptoms [23]. The proposed mechanism is that thinning of the endometrium by the therapy reduces bleeding and uterine contraction to ease menstrual pain [24]. Much like in therapies using phytoestrogens, soy (S)-equol [25] and isoflavones [26] were suggested to ameliorate PMS symptoms in crosssectional studies. In the study of (S)-equol, $10 \mathrm{mg}$ of (S)equol ameliorated a number of symptoms involved in PMS in Japanese female college students [27]. On the other hand, soy isoflavones $(20 \mathrm{mg}$ ) only suppressed "autonomic reaction" and "behavioral change" in MDQ scores of Korean American subjects in the crosssectional study [26]. Thus (S)-equol may be more effective than soy isoflavons and MPE; however; these studies were not placebo-controlled. Regarding (S)equol, a placebo-controlled trial is underway in Japan [27]. The result will be useful to compare the efficacy of MPE and (S)-equol. Compared with the studies of soy- derived compounds, the ameliorating effects of MPE on PMS clarified in the double-blind controlled study are more promising. More studies of the effects of soy isoflavones on postmenopausal syndromes have been reported than on their effects on PMS [29]. Phytoestrogens contained in MPE may be effective against postmenopausal syndromes, but further studies are required.

In blood analysis, MPE did not affect blood estradiol or progesterone (Table 8). Thus, the ameliorating effects of MPE are considered to be induced by the estrogenic effects of compounds in MPE [1] rather than indirect effects on the female hormone concentration. Recently in Japan, dietary supplements containing Pueraria candollei var. mirifica [16] for breast enlargement were reported to have abnormal effects in women around 20-years old, including abnormal bleeding. The side effects may have been induced by miroesterol and deoxymiroesterol, which exhibit strong estrogenic effects, similar to $17 \beta-$ estradiol [29]. However, there are no public organizations that can confirm the content of these compounds in P. candollei var. mirifica. The Japanese government implemented a new measure stating that the suppliers must report any incident of consumers having abnormal side effects. Therefore, safer estrogenic extracts containing phytoestrogens can be produced in Japan. This study did not note any abnormal changes in blood pressure, pulse, blood (Table 2), or urine parameters (Table 9) after MPE ingestion. There were no adverse effects related to menstruation or sexual organs. Therefore, ingestion of MPE was suggested to be safe and effective method to ease female anxieties related to menstruation. In conclusion, MPE was suggested to be a safe estrogenic extract that can ease several anxieties related to PMS in healthy women. 


\section{CONCLUSIONS}

This study demonstrated that MPE (100 mg/day for 8 weeks) ameliorated MDQ scores in healthy female subjects during the menstrual period. Therefore, MPE may be useful for improving monthly female anxieties. Furthermore, the intake of MPE was safe under the conditions of this study.

Abbreviations: ALP, alkaline phosphatase; ALT, alanine transaminase; AST, aspartate aminotransferase; BMI, body mass index; CK, creatinine kinase; GTP, glutamyltransferase; HDL, high-density lipoprotein; $\mathrm{Hb}$, hemoglobin; LAP, leucine aminopeptidase; LDH, lactate dehydrogenase; LDL, low-density lipoprotein; MCS, mental component summary; MDQ, menstrual distress questionnaire; MPE, moriche palm extract; MPE, mean cell volume; PCS, physical component summary; PMDD, premenstrual dysphoric disorder; PMS, premenstrual period; RCS, role/social component summary; SD, standard deviation; SF-36, Short-Form 36-Item

Competing Interests: The sponsor of the present study, Oryza Oil \& Fat Chemical Co., Ltd., assigned ORTHOMEDICO Inc. to conduct the study. S.T and H.S. (Ph.D.) are affiliated with Oryza Oil \& Fat Chemical Co., Ltd., and K.Y., N.S., S.Y., S.I., H.N., T.K., and A.S. are members of ORTHOMEDICO Inc. This study was conducted by both Oryza Oil \& Fat Chemical Co., Ltd. and ORTHOMEDICO Inc. T.T. (MD) was the principal investigator who monitored all the subjects' conditions. Furthermore, S.T., S.Y., and T.M.(Ph.D.) isolated and identified the chemical structure of 5,4'-hydroxy-7methoxy-6-methylflavan.

\section{Authors' Contributions: Conceptualization: H.S. and T.T.}

Data curation: H.N. and T.K. Formal analysis: T.K. Funding acquisition: H.S. Investigation H.N., T.K., A.B., and T.T. Methodology: K.Y., N.S., S.Y., S.I., and H.S.
Project administration and resources: K.Y., N.S., and T.T. Visualization: T.K. and A.B. Writing-original draft T.T. Writing-review and editing: K.Y., N.S., S.Y., S.I., H.N., T.H., A.B., and H.S. Quality evaluation of test sample: S.T., S.Y., and T.M.

Acknowledgements and Funding: This study was partially funded by a research and development grant from Aichi prefecture in 2018.

\section{REFERENCES}

1. Okamoto A, Ozawa T, Imagawa H, Arai Y: Flavans from the pith of sago palm. Agric. Biol. Chem. 1986, 50: 1655-1656.

2. Zheng QA, Li HZ, Zhang YJ, Yang CR: Flavonoids from the resin of Dracaena cochinchinensis. Helvetica Chim. Acta. 2004, 87: 1167-1171

3. Xu X, Cheng K, Cheng W, Zhou T, Jiang M, Xu J. Isolation and characterization of homoisoflavonoids from Dracaena cochinchinensis and their osteogenic activities in mouse mesenchymal stem cells. J. Pharm. Biomed. Anal. 2016, 129, 466-472.

4. Zhan G, Zhou J, Liu T, Zheng G, Aisa HA, Yao G: Flavans with potential anti-inflammatory activities from Zephyranthes candida. Bioorg. Med. Chem. Lett. 2016, 26: 5967-5970.

5. Wu JH, Tung YT, Chien SC, Wang SY, Kuo YH, Shyur LF, Chang ST: Effect of phytocompounds from the heartwood of Acacia confusa on inflammatory mediator production. J. Agric. Food Chem. 2008, 56: 1567-1573.

6. Pathak V, Shirota O, Sekita S, Hirayama Y, Hakamata Y, Hayashi T, Yanagawa T, et al.: Antiandrogenic phenolic constituents from Dalbergia cochinchinensis. Phytochemistry. 1997, 46: 1219-1223.

7. Ahn JH, Liu Q, Lee C, Ahn MJ, Yoo HS, Hwang BY, Lee MK: A new pancreatic lipase inhibitor from Broussonetia kanzinoki. Bioorg. Med. Chem. Lett. 2012, 22: 2760-2763.

8. Liao XL, Luo JG, Kong LY: Flavonoids from Millettia nitida var. hirsutissima with their anticoagulative activities and inhibitory effects on NO production. J. Nat. Med. 2013, 67: 856-861.

9. Qiao M, Zhang H, Yu Y, Ci Y, Xu X, Ye Q, Chen Y. Dynamic changes in serum estradiol and progesterone levels in patients of premenstrual syndrome with adverse flow of 
liver-qi. J. Tradit. Chin. Med. 2008, 28: 106-109.

10. Verdonk SJE, Vesper HW, Martens F, Sluss PM, Hillebrand JJ, Heijboer AC. Estradiol reference intervals in women during the menstrual cycle, postmenopausal women and men using an LC-MS/MS method. Clin. Chim. Acta. 2019, 495: 198-204.

11. Sathyapalan T, Aye M, Rigby AS, Thatcher NJ, Dargham SR, Kilpatrick ES, Atkin SL: Soy isoflavones improve cardiovascular disease risk markers in women during the early menopause. Nutr. Metab. Cardiovasc. Dis. 2018, 28 691-697.

12. Zheng X, Lee SK, Chun OK: Soy isoflavones and osteoporotic bone loss: A review with an emphasis on modulation of bone remodeling. J. Med. Food. 2016, 19: 1-14.

13. Davinelli S, Scapagnini G, Marzatico F, Nobile V, Ferrara N Corbi G: Influence of equol and resveratrol supplementation on health-related quality of life in menopausal women: A randomized, placebo-controlled study. Maturitas. 2017, 96: 77-83.

14. Bryant M, Cassidy A, Hill C, Powell J, Talbot D, Dye L: Effect of consumption of soy isoflavones on behavioural, somatic and affective symptoms in women with premenstrua syndrome. Br. J. Nutr. 2005, 93: 731-739.

15. Rahimikian F, Rahimi R, Golzareh P, Bekhradi R, Mehran A: Effect of Foeniculum vulgare Mill. (fennel) on menopausal symptoms in postmenopausal women: a randomized, triple-blind, placebo-controlled trial. Menopause. 2017, 24:1017-1021

16. Kongkaew C, Scholfield NC, Dhippayom T, Dilokthornsakul P, Saokaew S, Chaiyakunapruk N: Efficacy and safety of Pueraria candollei var. mirifica (Airy Shaw \& Suvat.) Niyomdham for menopausal women: A systematic review of clinical trials and the way forward. J. Ethnopharmacol. 2018, 216:162-174.

17. Wong RHX, Evans HM, Howe PRC: Resveratrol supplementation reduces pain experience by postmenopausal women. Menopause. 2017, 24:916-922.

18. Shamshad Begum S, Jayalakshmi HK, Vidyavathi HG, Gopakumar G, Abin I, Balu M, Geetha K, et al.: A novel extract of fenugreek husk (FenuSMART ${ }^{\mathrm{TM}}$ ) alleviates postmenopausal symptoms and helps to establish the hormonal balance: A randomized, double-blind, placebo- controlled study. Phytother. Res. 2016, 30:1775-1784.

19. Matsuda H, Shimoda H, Morikawa T, Yoshikawa M: Phytoestrogens from the roots of Polygonum cuspidatum (Polygonaceae): Structure-requirement of hydroxyanthraquinones for estrogenic activity. Bioorg Med Chem Lett 2001, 11: 1839-1942.

20. Moos RH: The development of a menstrual distress questionnaire. Psychosom. Med. 1968, 30: 853-867.

21. Suzukamo Y, Fukuhara S, Green J, Kosinski M, Gandek B, Ware J: Validation testing of a three-component model of Short Form-36 scores. J. Clin. Epidemiol. 2011, 64: 301-308.

22. Chung SH, Kim TH, Lee HH, Lee A, Jeon DS, Park J. Kim Y. Premenstrual syndrome and premenstrual dysphoric disorder in perimenopausal women. J. Menopausal Med. 2014, 20: 69-74

23. Lopez LM, Kaptein AA, Helmerhorst FM: Oral contraceptives containing drospirenone for premenstrual syndrome. Cochrane Database Syst. Rev. 2012, 2: CD006586.

24. Takahashi T: Menstrual symptoms. Clinical Gynetrogy Obstetrics 2010, 71, 63-73 (in Japanese)

25. Takeda T, Ueno T, Uchiyama S, Shiina M: Premenstrual symptoms interference and equol production status in Japanese collegiate athletes: A cross-sectional study. J. Obstet. Gynaecol. Res. 2018, 44: 488-494.

26. Kim HW, Kwon MK, Kim NS, Reame NE. Intake of dietary soy isoflavones in relation to perimenstrual symptoms of Korean women living in the USA. Nurs Health Sci. 2006, 8: 108-113.

27. Takeda T, Shiina M, Chiba Y: Effectiveness of natural S-equol supplementation for premenstrual symptoms: protocol of randomized, double-blind, placebo-controlled trial. $B M J$ Open 2018, 8: e023314.

28. Martín Salinas C, López-Sobaler AM: Benefits of soy in women's health. Nutr Hosp. 2017, 34 (Suppl 4): 36-40

29. Matsumura A, Ghosh A, Pope GS. Darbre PD: Comparative study of estrogenic properties of eight phytoestrogens in MCF7 human breast cancer cells. J. Steroid Biochem. Mol. Biol. 2005, 94: 431-443. 